\title{
Smart Intersection Management system
}

\author{
Shaikh Mohd Rahil ${ }^{1}$, Shreelaxmi ${ }^{2}$, Suhas Meti ${ }^{3}$, Vishwanath G.N. ${ }^{4}$, Ganesh Shetty ${ }^{5}$ \\ UG Student, Electronics \& Communication Department, Shri Madhwa Vadiraja Institute of Technology \&
}

Management, Udupi, India ${ }^{1,2,3,4}$

Assistant Professor, Electronics \& Communication Department, Shri Madhwa Vadiraja Institute of Technology \&

Management, Udupi, India ${ }^{5}$

\begin{abstract}
The congestion of the urban traffic is becoming one of the critical issues with increasing population and automobiles in cities. Traffic jams not only cause extra delay and stress for the drivers, but also increase fuel consumption, add transportation cost, and increase carbon dioxide air pollution. The traffic controller is one of critical factors affecting the traffic flow. The conventional traffic patterns are nonlinear and complex and time dependent rather than traffic dependent. Here we are proposing a traffic based feedback intersection system which changes the time of green and red light with respect to the traffic flow rather than time. The "SIMS" utilizes very basic hardware to ensure that costs are reasonable \& the maintenance is low. The entire project uses Image processing as its principle. The hardware used are Camera, Stepper Motor, Raspberry Pi, Arduino Uno, LCD, LED. This solution to the ever increasing urbanization would help in lowering the level of traffic congestion in many large cities, like Bangalore which experiences a very high level of traffic congestion that has been unprecedented. The result is that most road networks in urban areas would provide an optimal transport system and would also reduce the air pollution, fuel consumption and various other negative factors. Also it would ensure a reduction in accidents due to inefficient traffic management.
\end{abstract}

Keywords: nonlinear, traffic dependent, Image processing, Raspberry Pi, Arduino Uno, traffic congestion.

\section{INTRODUCTION}

With the rapid growth of industrialization, urbanization, population a tremendous growth has occurred in the number of vehicles, leading to the increased traffic. It further causes traffic rule violation, heavy traffic signals, accidents, traffic jam which also causes a lot of wastage of time. The traffic lights established these days are static i.e. they are unable to adjust the time duration of the signals according to the density of the lane as well as unable to prioritise the emergency vehicles. Sometimes the vehicles on the red light lane have to wait for green signal even though there is no traffic where the green signal is allotted. It results in the loss of valuable time as well as loss in fuel consumption resulting pollution. Travelling to different places within the city is becoming more difficult, there is a loss in productivity from workers, people loose time; trade opportunities are lost, delivery gets delayed, and thereby the costs goes on increasing which ultimately leads to frustration and imbalanced life. Therefore many countries are working on efficiently managing their existing transportation systems to render improved mobility and safety. Hence an automatic traffic management scheme which works on real time traffic is becoming prominent. The simplest form of traffic management includes human in the system. In this scheme, a traffic officer is placed on each and every cross-section of the roads; the traffic police control the flow of traffic. In time of congestion she/he gives the signals to the vehicle driver whether to stop or drive. She/he is also able to recognise emergency case, so she/he can choose which lane needs more priority than other. This scheme is most efficient than any other technique. But as it includes human as a part of system this scheme is inadequate. Efficiency of system depends on experience and capability of the person.

In order to remove the weak link i.e. human in above system, an automatic traffic management scheme is suggested. The system which we see every day includes simple three colour traffic signal. Generally for each lane 120 seconds of green light is on. Before green light, yellow light flashes for 20 second, signifying to start the vehicle and be ready to go. For all the time red light is on, indicating each vehicle to stop. This system cannot identify emergency vehicle, it treats normal car and ambulance in the same way. So there are chances of delay in the emergency services.

\section{OBJECTIVE}

Currently the traffic control system in place in our country is non-flexible and non-adaptive to the ever growing number of vehicles on the road. It does not take into account the changing density of traffic during the different hours of the day. Consequently, the roads get congested frequently and intersections get blocked.

Time and fuel, two highly important resources get wasted in this inefficient working of the present-day system. In this article, we propose a dynamic system that overcomes all these drawbacks. 
- Our system uses cameras installed at the red lights and intersections to monitor the traffic dynamically.

- It then processes this information using image processing,

- Computes the volume of the real time traffic, sets the timer of the signal accordingly.

- Simultaneously, it monitors if there is any scope of congestion at the intersection and adjusts the timer to prevent it. The entire system works autonomously and has a quick turnaround time, saving critical resources at every junction.

\section{III.LITERATURE REVIEW}

In [1], the proposed system makes use of the background differencing of an empty lane road. The resulting output is fed to the microcontroller system for traffic light control system on real time data.

In [2], a method of real time area based traffic estimation; using image processing for intelligent traffic control system is described. This paper makes use of edge detection. The more the vehicles in a road, the more is the area of the edges of the vehicles which means the traffic congestion is more. Thus more duration can be allotted for that road.

In [3], a method is proposed for determining traffic congestion on roads using image processing techniques and a model for controlling traffic signals based on information received from images of roads taken by video camera. Here the empty road will be the background image and subsequent frames from the video camera will be the foreground image. By subtracting background image from the foreground image we can find out traffic density present in a frame. In [4], the system will detect vehicles through images instead of using electronic sensors embedded in the pavement. A camera will be installed alongside the traffic light. It will capture image sequences. Setting image of an empty road as reference image, the captured images are sequentially matched using image matching. For this purpose, edge detection has been carried out using Prewitt edge detection operator and according to percentage of matching traffic light durations can be controlled.

\section{IV.METHODOLOGY}

RGB coloured image are captured using the camera and sent to the processing unit. There it is converted into a gray scale image which undergoes binary conversion. The image being binary image, it either has pixels with value of 255 or 0 , no grey levels. The image is then processed to calculate the white and the black area which is used to calculate the density of the traffic. The calculations are then used to assign sequences to switch the light. Figure 1 shows the basic diagram of the proposed system.

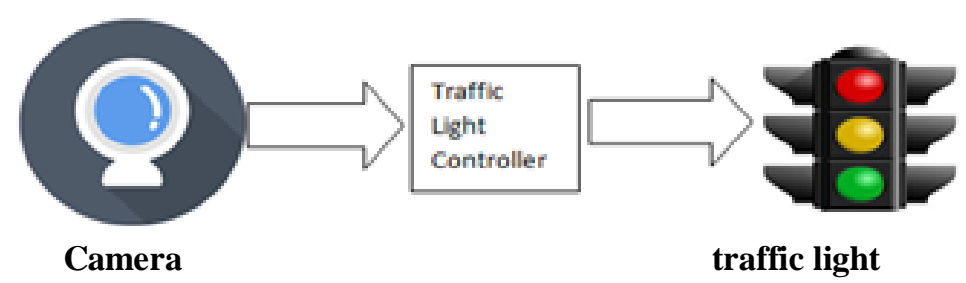

Fig 1: Basic diagram

A. Block diagram:

Figure 2 shows the block diagram of the proposed model. The whole model is placed at the intersection of 4 traffic lanes: lane 1, lane 2, lane 3 and lane 4.

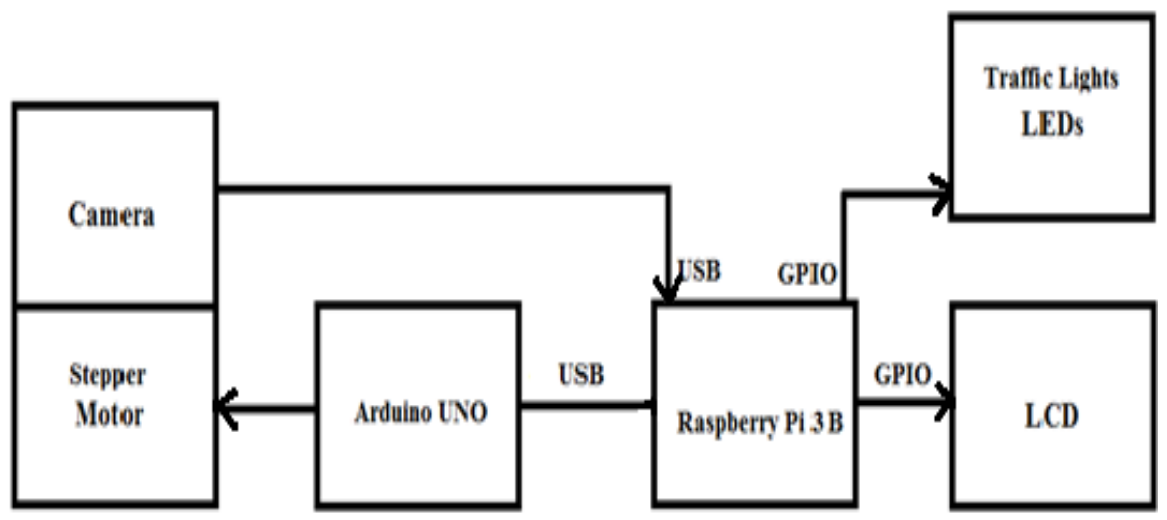

Fig 2: Block diagram 


\section{ISO 3297:2007 Certified}

Vol. 5, Issue 6, June 2017

The 8 megapixel camera is placed on the NEMA 17 stepper motor. The stepper motor is programmed with Arduino code. The Arduino Uno and the Raspberry pi 3B v1.2 module are serially connected using USB cable for synchronization of operation between the camera and the stepper motor. The camera captures the image of a particular traffic lane and sends the signals to the Raspberry Pi through USB cable. Raspberry Pi processes the signals using the code in Python language. Python is used because it has many built in libraries to process the image and the standard English words can be used for coding.

The Raspberry Pi performs the following basic steps with the help of Python code:

1. The first step is to get the Traffic length $\left(T_{\text {Len }}\right)$ and traffic density $\left(T_{\text {Dens }}\right)$ using image processing techniques like:

- Cropping: The photo captured from the camera is cropped for the required region of the road.

- Image segmentation: It is used for partitioning the digital image into multiple segments (sets of pixels known as super pixels) thus locating the vehicles and their boundaries (lines, curves, etc.).

The result of image segmentation is a set of segments that collectively cover the entire image, or a set of contours extracted from the image. Each of the pixels in a region is similar with respect to properties such as colour, intensity, or texture. Adjacent regions are significantly different with respect to the same characteristics [5].

- $\quad$ Filtering: It is a technique for modifying or enhancing an image to emphasize certain features or remove other features. Image processing operations implemented with filtering include smoothing, sharpening, and edge enhancement [6].

- Otsu's thresholding: This is used to automatically perform clustering based image thresholding or the reduction of a gray level image to a binary image. It is done by using numpy library in Python. In global thresholding an arbitrary value is taken as threshold value. If pixel value is greater than a threshold value, it is assigned one value (may be white), else it is assigned another value (may be black).In Otsu binarization, bimodal image is considered. It is an image whose histogram has two peaks. For that image, an approximate value in the middle of those peaks is taken as a threshold value. Otsu's thresholding automatically calculates a threshold value from image histogram for a bimodal image. Figure 3 represents global thresholding with noisy image, Otsu's thresholding with noisy image and Otsu's thresholding with Guassian filtered image [7].

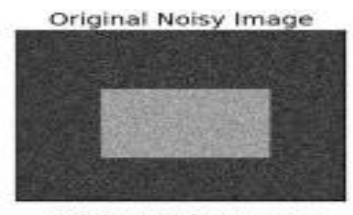

Original Noisy Image

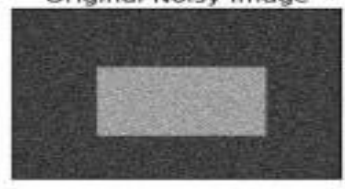

Gaussian filtered image

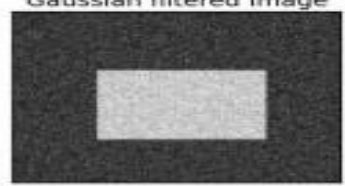

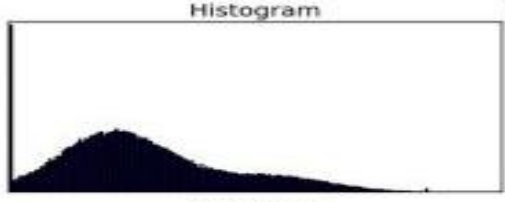

Histogram

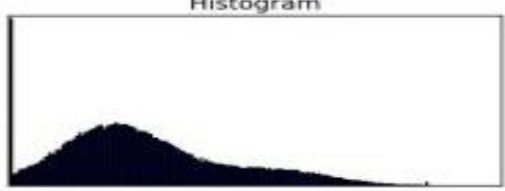

Histogram

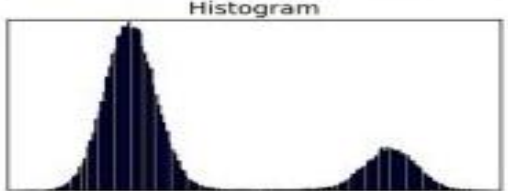

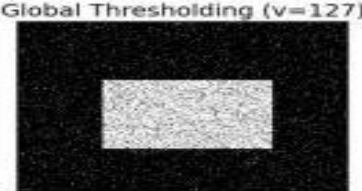

Otsu's Thresholding

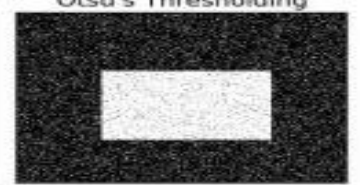

Otsu's Thresholding

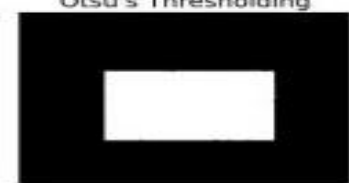

Fig 3: global thresholding with noisy image, Otsu's thresholding with noisy image and Otsu's thresholding with Guassian filtered image

- Counting the number of white pixels out of the total number of pixels and counting the number of objects using edge detection.

2. The second step is to use the data collected above to change the time of the traffic signals as green for a specific direction of traffic at an intersection.

3. Wait for a time duration $\mathrm{T}_{\mathrm{dur}}$ that is a dynamic value, calculated according to the traffic conditions in the allowed directions. The maximum permissible period for one pair of directions is fixed (at $T_{\max }$ ) but can be reduced to decide $\mathrm{T}_{\mathrm{dur}}$ as the traffic in the allowed direction decreases.

4. The amount of time that a signal turns green is decided by the following equation:

$$
\mathrm{T}_{\mathrm{dur}}=\mathrm{T}_{\text {max }}-\left(\mathrm{T}_{\text {max }} * \mathrm{k}\right)
$$




\section{International Journal of Innovative Research in} Electrical, Electronics, Instrumentation and Control Engineering

\section{ISO 3297:2007 Certified}

Vol. 5, Issue 6, June 2017

Where $\mathrm{k}$ is decided from the equation

Where

$$
\mathrm{k}=\text { normalized density }+ \text { count }
$$

Normalized density $=$ calculated density/maximum density

B. Timing Diagram:

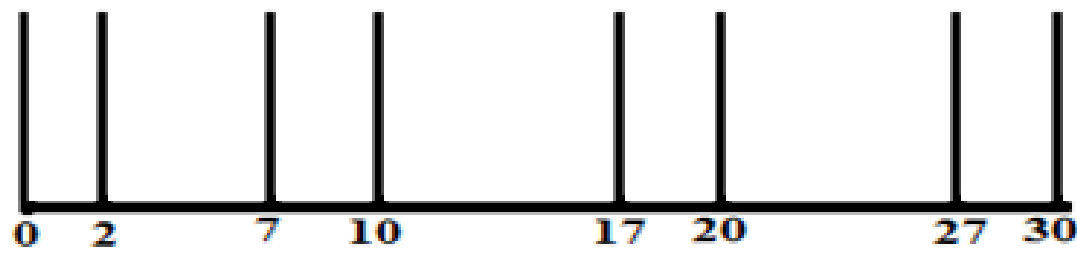

Fig 4: Sequence of operation within 30 seconds of time
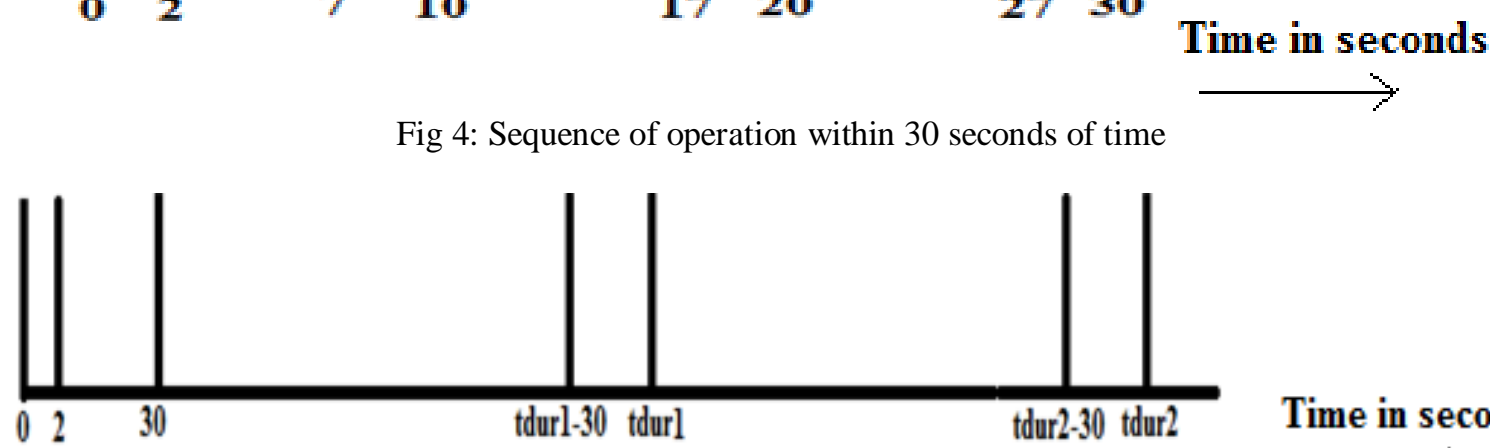

Time in seconds

Fig 5: Timing Diagram of LED light control

Steps:

In figure 4, the sequence of operation within first 30 seconds of time is shown.

At 0 , Lane 1 photo is taken.

At 2, Stepper motor rotates 90 degree anticlockwise.

At 7, Lane 2 photo is taken.

At 10, Stepper motor rotates 90 degree anticlockwise.

At 17, Lane 3 photo is taken.

At 20, Stepper motor rotates 90 degree anticlockwise.

At 27, Lane 4 photo is taken.

At 30, Stepper motor rotates back to its initial position clockwise.

In figure 5 , the entire process flow is shown.

1. At 0 , all the 4 lanes will have traffic light as red. The camera initially faces towards lane 1 .

2. Between 0 and 2, the camera captures the photo of the traffic in lane 1 .

3. At 2, the stepper motor connected to the Arduino Uno rotates by 90 degree to face the lane 2 traffic. Similarly, the camera and the stepper motor operate to capture the pictures of lane 2, lane 3 and lane 4 traffic respectively, as per the sequence of steps mentioned for figure 4.

4. At 30, the captured images are given to the Raspberry pi 3 B v1.3 module through USB cable. The Raspberry Pi module processes the captured images and the resulting signals are passed to the LED and LCD display through GPIO pins of the Pi module. The LED shows the green signal for the lane with maximum traffic for $T_{\text {dur }}$ time and red signal for rest of the lanes. $T_{\text {dur }}$ can be in between $T_{\max }=120$ seconds or $T_{\min }=35$ seconds of time. In the first case, $T_{\text {dur }}=T_{\text {durl. }}$. The $L C D$ acts as a timer and shows the countdown values from $T_{\text {durl }}$ to 0 .

5. $t_{d u r}-30$, The procedure for the evaluation of the next traffic scenario should start before 30 seconds of the completion of $\mathrm{T}_{\text {durl }}$. Therefore the stepper motor starts to rotate and the camera starts to capture the photos again.

6. At $t_{\text {durl }}$, for the first set of images, the green signal to the maximum traffic lane ends in LED and timer stops in LCD. The Raspberry Pi evaluates the maximum traffic lane for the second set of images and gives the green signals to $\mathrm{LED}$ for $\mathrm{T}_{\text {dur }}$ time and the $\mathrm{T}_{\mathrm{dur} 2}$ time for $\mathrm{LCD}$.

7. At $t_{\text {dur } 2}-30$, The stepper motor runs and the photos are taken for next 30 seconds for the $3^{\text {rd }}$ set of traffic scenario.

8. At $t_{\text {dur }}$, for the second set of images, the green signal to the maximum traffic lane ends in LED and timer stops in LCD. The Raspberry Pi evaluates the maximum traffic lane for the second set of images and gives the green signals to $\mathrm{LED}$ for $\mathrm{T}_{\text {dur3 }}$ time and the $\mathrm{T}_{\mathrm{dur} 3}$ time for $\mathrm{LCD}$ and the process continues. 


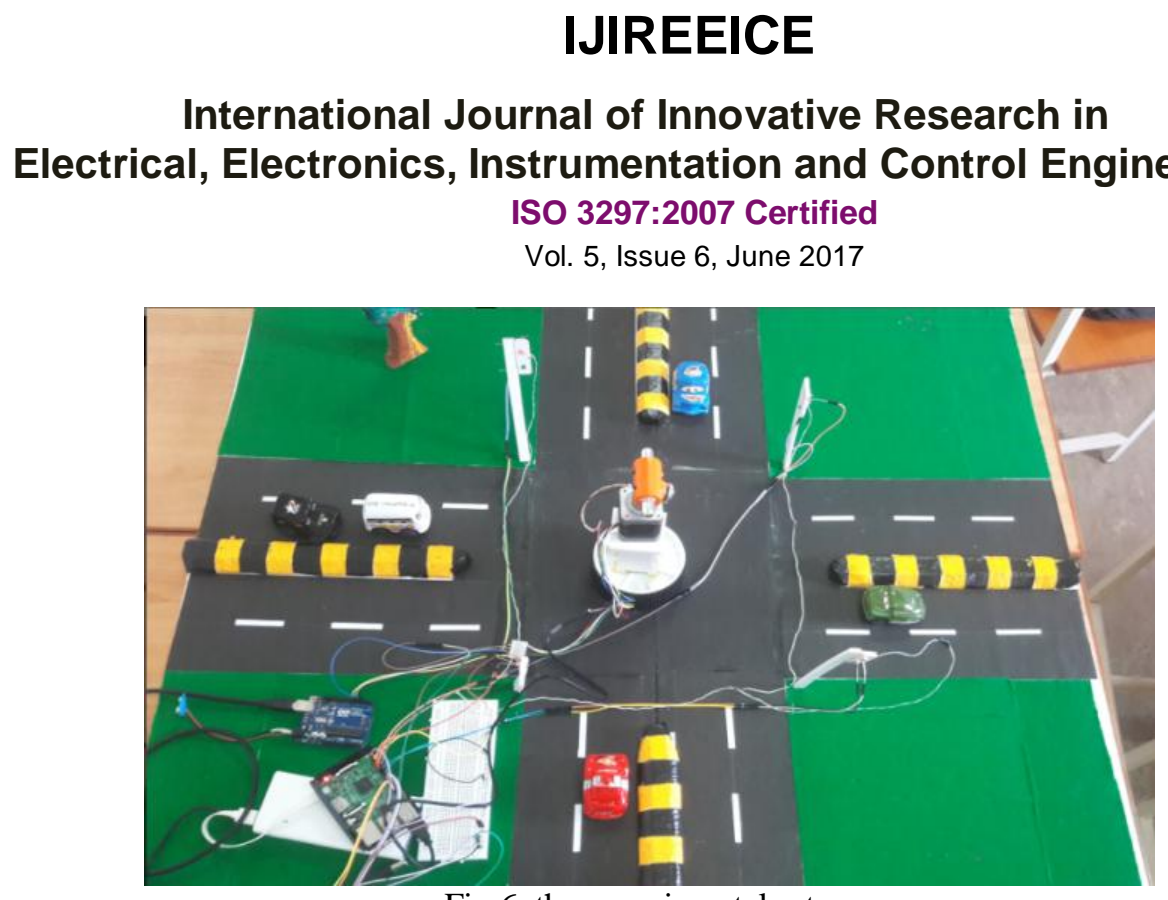

Fig 6: the experimental setup

\section{ADVANTAGES \& DISADVANTAGES}

A. Advantages

The following are the advantages of the proposed system:

- By alternately assigning right of way to various traffic movements, signals provide for the orderly movement of conflicting flows. Thus efficient management of the traffic in the intersection can be achieved.

- The time being wasted in automatic traffic control by providing green light on the empty road is avoided.

- avoids traffic jams

- The resources such as petrol, diesel and natural gas can be saved.

- The optimization will lead to faster transportation of goods.

- Cameras installed can be used for security purposes.

- Avoids accidents

B. Disadvantages

There are some demerits in the proposed system:

- The system is efficient up to $80 \%$ as the system has difficulty to detect black coloured vehicles.

- Requires high definition camera

\section{RESULTS AND DISCUSSIONS}

The images of traffic in lane 1, lane 2, lane 3 and lane 4 are captured using the camera mounted over the stepper motor at the intersection. Figure 6, figure 7, figure 8 and figure 9 show the captured image of lane 1, lane 2, lane 3, lane 4 in the camera's view respectively.

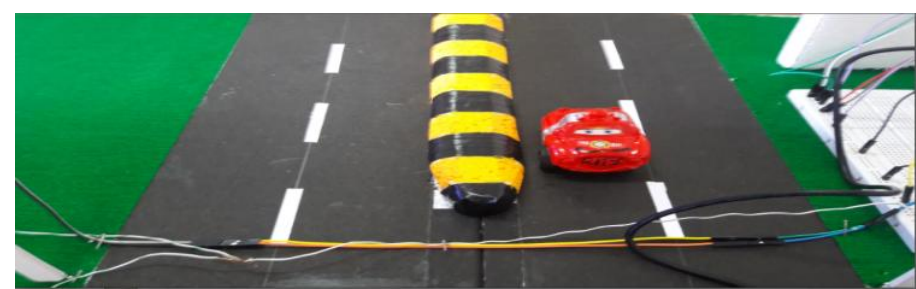

Fig 6: Lane 1 image

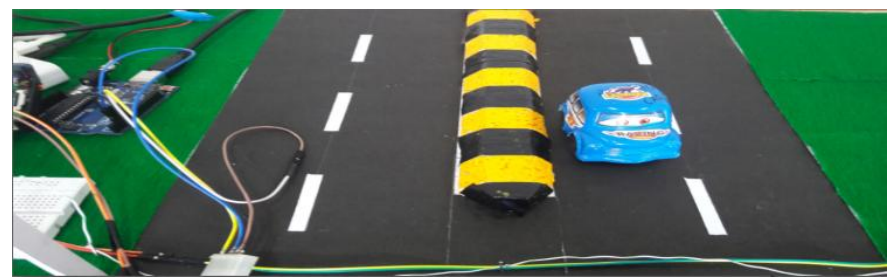

Fig 7: Lane 2 image 
IJIREEICE

International Journal of Innovative Research in Electrical, Electronics, Instrumentation and Control Engineering

ISO 3297:2007 Certified

Vol. 5, Issue 6, June 2017

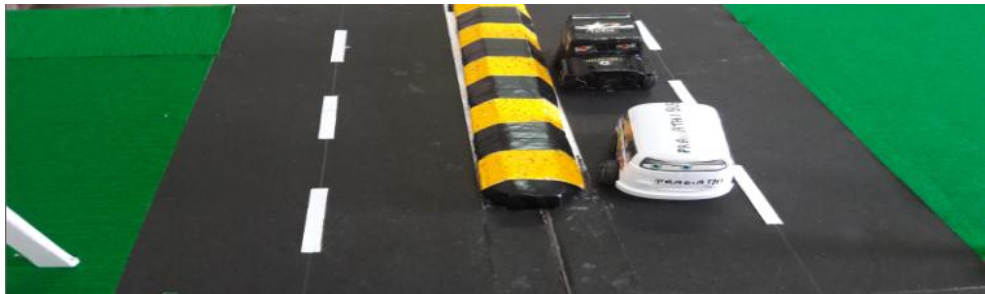

Fig 8: Lane 3 image

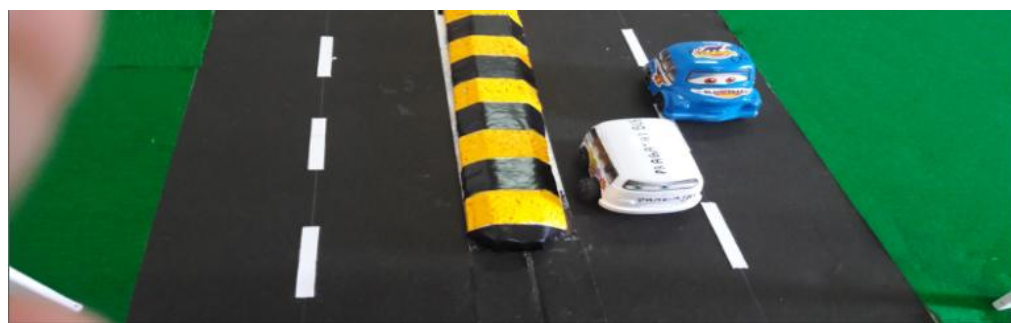

Fig 9: Lane 4 image

The segmented-images of traffic in lane 1, lane 2, lane 3 and lane 4 are as shown in figure 10, figure 11, figure 12 and figure 13 respectively.
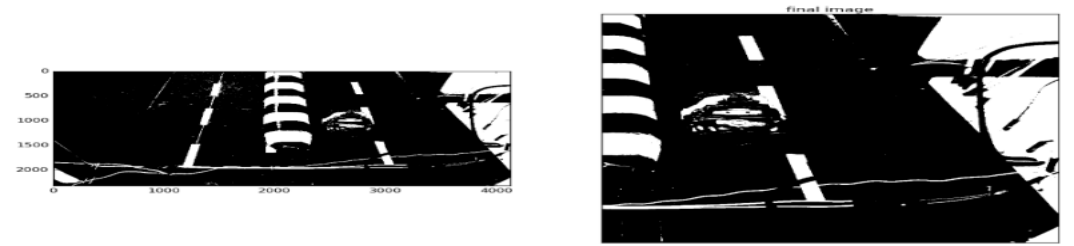

Fig 10: Lane 1 segmented image
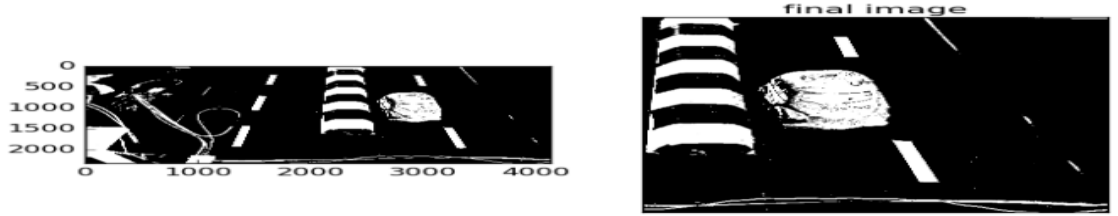

Fig 11: Lane 2 segmented image
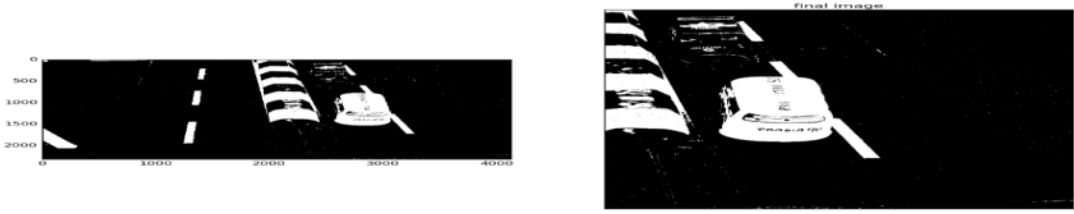

Fig 13: Lane 3 segmented image
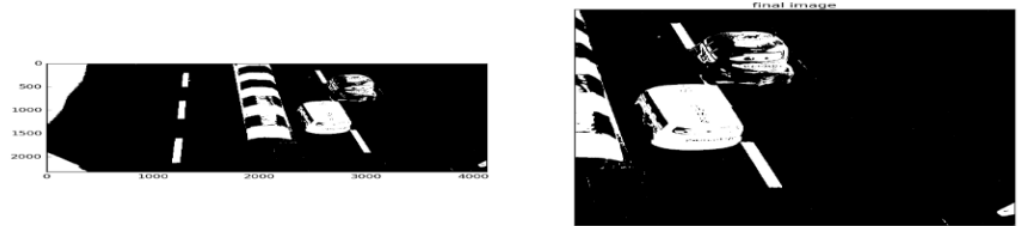

Fig 14: Lane 4 segmented image

Figure 15 shows the simulation result after image processing for the images in figure 11, 12, 13 and 14. 


\section{International Journal of Innovative Research in Electrical, Electronics, Instrumentation and Control Engineering}

ISO 3297:2007 Certified

Vol. 5, Issue 6, June 2017

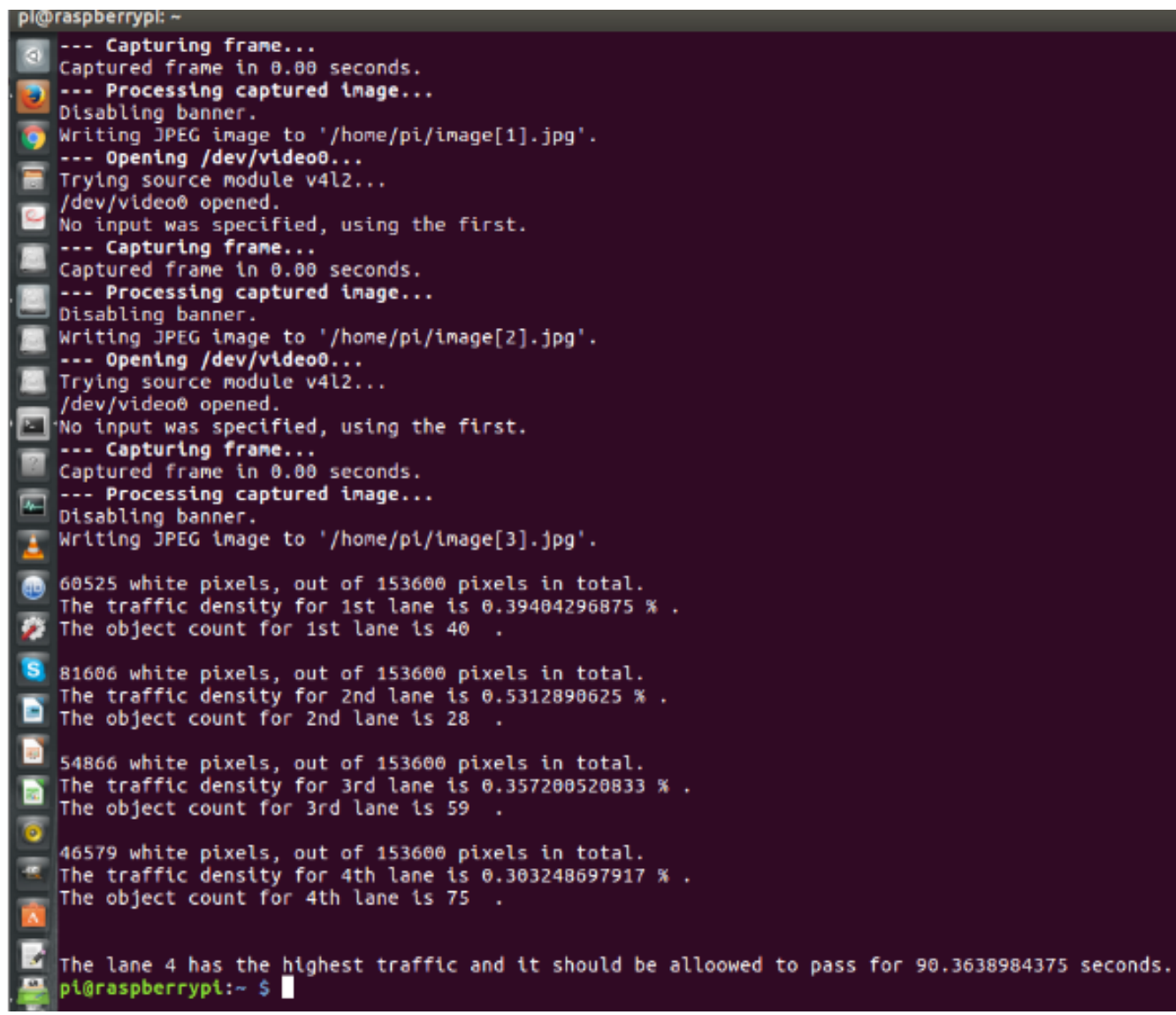

Fig: 15 Simulation Result

\section{CONCLUSION}

The proposed traffic control system is adaptive for the dynamic and real time traffic. It can be used to easily determine the traffic density according to the simulations so far. This area based traffic system can be used in densely populated areas where the number of automobiles is increasing gradually. Our system will play an important role in estimating traffic congestion to control traffic signals but it can still be improved by

- $\quad$ including features such as emergency vehicle detection in the case of ambulance

- $\quad$ using better algorithms

- learning the traffic hours.

\section{ACKNOWLEDGMENT}

The student authors would like to thank Dr. Balachandra Achar H V, Professor and Head and Mr. Ganesh Shetty, Assistant Professor, Department of Electronics and Communication Engineering, SMVITM, Udupi for their exemplary guidance, monitoring and constant encouragement throughout the completion of our final year B.E project.

We also express a deep sense of gratitude to thank Dr. Thirumaleshwara Bhat, Principal, SMVITM, Udupi for providing well organised infrastructure for the completion of our project.

\section{REFERENCES}

[1] Sarpong Kwadwo Asare and Robert A. Sowah, Department of computer Engineering, "Design and development of a Microcontroller Based Traffic Control System Using Image Processing Techniques:- A case study prototype for Legon - Okponglo Junction, University of Ghana."

[2] Mohammad Shahab Uddin1, Ayon Kumar Das2, Md. Abu Taleb, Department of Electrical and Electronic Engineering, Chittagong University of Engineering and Technology Chittagong, Bangladesh "Real-time Area Based Traffic Density Estimation by Image Processing for Traffic Signal Control System: Bangladesh Perspective" 2nd Int'l Conf. on Electrical Engineering and Information \& Communication Technology (ICEEICT) 2015 Jahangirnagar University, Dhaka-1342, Bangladesh, 21-23 May

[3] Pranav Maheshwari, Praneet Singh, Deepanshu Suneja, Yogeshwar Mutneja "Smart Traffic Optimization Using Image Processing", IlCA, Volume 118 - Number 23, 2015.

[4] Ms Pallavi Chowdekar, Ms Sayanti Banerjee, Prof. MK Muju "Implementation of image processing in real time traffic light control" 
[5] [Online]https://en.wikipedia.org/wiki/Image_segmentation

[6] [Online]http://in.mathworks.com/help/images/what-is-image-filtering-in-the-spatial-domain.html

[7] [Online] http://docs.opencv.org/trunk/d7/d4d/tutorial_py_thresholding.html

\section{BIOGRAPHIES}

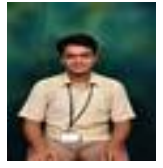

Shaikh Mohd Rahil currently pursuing the Bachelor's degree in Electronics \& Communication Engineering from Shri Madhwa Vadiraja Institute of Technology \& Management, Udupi, Karnataka.

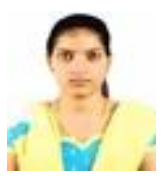

Shreelaxmi currently pursuing the Bachelor's degree in Electronics \& Communication Engineering from Shri Madhwa Vadiraja Institute of Technology \& Management, Udupi, Karnataka.

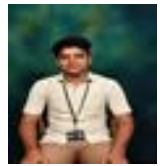

Suhas M. Meti currently pursuing the Bachelor's degree in Electronics \& Communication Engineering from Shri Madhwa Vadiraja Institute of Technology \& Management, Udupi, Karnataka.

Vishwanath G.N. currently pursuing the Bachelor's degree in Electronics \& Communication Engineering from Shri Madhwa Vadiraja Institute of Technology \& Management, Udupi, Karnataka.

Mr. Ganesh Shetty received the B.E. degree in Instrumentation Technology from Malnad College of Engineering Hassan, in the year 2008, and M.Tech. in Industrial Electronics from SJCE, Mysore, India, in 2013. He is currently working as Assistant Professor of E\&C Engineering at SMVITM. He is in the field of teaching for 6 years. 